\title{
Evaluation of a Single Oncoplastic Technique for Peri-Areolar Upper Quadrants Breast Cancer
}

\author{
Mostafa M. Elghalban, Mohamed Ghazaly, Ahmed A. Darwish, Ayman A. Elnemr, \\ Waleed Y. El Sherpiny
}

Oncology Unit, Department of Surgery, Tanta University, Tanta, Gharbia, Egypt

\section{ABSTRACT}

Background: Oncoplastic surgery has gained wide popularity. Batwing mastopexy (BWM) is an oncoplastic technique being used in treatment of centrally located tumors. The purpose of our study is to assess short-term outcome including cosmetic results of this technique done for peri-areolar lesions in breast upper quadrants.

Methods: A prospective study of a series of 25 women with upper quadrants peri-areolar breast cancer. 23 patients underwent wide local excision with clear margins accompanied by BWM in order to achieve pleasant outcome. Separate incisions were performed for axillary biopsy or dissection for invasive cancers. Four months after surgery, the cosmetic result was evaluated both objectively by the surgeons (five elements of the ABNSW system) and subjectively by patients regarding their satisfaction.

Results: Results were rated as poor, fair, good or excellent. This revealed good result in 18 cases $(78 \%)$ followed by fair in 4 cases $(17 \%)$. Again, most patients $18(78 \%)$ expressed good satisfaction with none of them reporting poor outcome either objectively by surgeons or even subjectively. Minor complications occurred in only 5 patients (21.7\%), all have been treated conservatively.

Conclusion: Batwing mastopexy is a safe, convenient-to-learn, and quick technique. It allows to achieve a favorable breast shape, particularly in women with large breasts.

Key words: early breast cancer, oncoplastic surgery, breast conservative treatment, mastopexy

\section{INTRODUCTION}

Breast cancer is a global challenge, and its treatment has evolved dramatically recently. The task of surgeons is not limited only to surgical resection of the lesion. They are the first contacts of the patient, members of a multidisciplinary team, leading the patient through the various diagnostic and therapeutic modalities of current breast cancer management (1). Few decades ago, surgical management for early breast cancer was modified radical mastectomy. Since then, randomised studies have demonstrated that breast-conservative surgery (BCS) with radiotherapy provides outcomes similar to those achieved by mastectomy in terms of survival (2). These outcomes have led to the emergence of BCS as the modality of choice for
Corresponding author: Mohamed Ghazaly, MD Department of Surgery Tanta University Hospitals El-Gaish, Tanta Qism 2, Tanta Gharbia Governorate, 31512, Egypt Phone: 00201112137427

E-mail: mohamed.ghazaly@med.tanta.edu.eg
Received: 23.12.2020 Accepted: 07.03.2021 
patients with early breast cancer, unless there is a contraindication to BCS or it's excluded by the patient (3). However, there is still a conflict between obtaining optimal tumor resection and not removing too much tissue that might lead to breast disfigurement, the indications for breast conserving surgery (BCS) in cancer are increasing. If a significant resection is required or if there is a high risk of deformity, if the breast shape and appearance are to be maintained, it may be difficult to achieve reliable local disease control (4).

In the last 2 decades, oncoplastic surgery has expanded dramatically, gaining widespread popularity, and is now highly recommended by well established guidelines (5). It allows comprehensive resections without jeopardizing the cosmetic outcome (6). The best option of oncoplastic alternatives depends on the size and location of the tumor besides the shape and volume of the breast. In certain patients, there would be a need for bilateral breast reduction by various techniques of rotation and repositioning of the nippleareola complex (NAC) but simpler procedures for decent cosmetic results are also possible, i.e. unilateral oncoplastic surgery without contralateral breast symetrisation (7). Among these methods is batwing mastopexy (BWM), which is being used for centrally located tumors (8).

The aim of our study was to assess the short-term outcome including cosmetic results of batwing mastopexy performed for peri-areolar malignant lesions located in the upper quadrants of breast.

\section{PATIENTS AND METHODS}

This study was conducted prospectively on 25 female patients presented with breast cancer in the upper quadrants whom were managed and treated at the surgical oncology unit at Tanta University Hospital during the period from October 2015 to October 2017.

Inclusion criteria: all patients presented \& diagnosed to have their lesions located in peri-areolar upper quadrants that can be managed by conservative breast surgery. Exclusion Criteria; multicentric cancers, tumor invasion of chest wall and skin, metastatic breast cancer, breast lumps larger than $4 \mathrm{~cm}$, and lumps far away from areola more than $3 \mathrm{~cm}$.

Evaluation criteria: all patients were subjected to the following: pre-operative work up including explanation and an informed consent before admission including consent for clinical photography.

Full history and clinical examination: local and systemic that include breast examination (all quadrants), mass examination (site, size, number, consistency, skin overlying and mobility), nipple and areola examination, and axillary examination. Investigations included routine laboratory workup: complete blood count, liver function tests, virology, renal function tests, fasting and postprandial blood glucose level and coagulation profile, tumor Markers: Cancer antigen 15-3 (CA 15-3) and Carcinoembryonic antigen (CEA). Imaging studies included breast ultrasound, mammogram, chest $x$-ray and abdominal and pelvic ultrasound. Fine Needle Aspiration Cytology (FNAC) or tru-cut biopsy was done to all patients to confirm the diagnosis.

Cardiac, chest and anaesthetic consultation before undergoing the surgical procedure.

Marking and photography: in the operative theatre with the patient in the upright position.

Anesthesia and patient positioning: the procedure was performed under general anesthesia. Patient was positioned on the operating room table in a supine position and the arm of the operative side was abducted at right angle on an arm board. The surgical area was disinfected by application of $10 \%$ povidone-iodine (betadine).

\section{Operative Techniques}

The incision was designed by placing two circumferentially arranged incisions that were parallel to the upper areolar border, the lower one located at the upper border of NAC and arranged circumferentially along this border, which often involves half the NAC circumference. The upper incision, parallel to the lower incision, was then made with a gap between these incisions varying from 3 to $5 \mathrm{~cm}$, depending on the lesion size, with a macroscopic margin of at least $1 \mathrm{~cm}$ of normal tissue. Two additional lateral triangular incisions with their apices directed peripherally away from the NAC were then made starting from the ends of the circumferential incisions. Depending on the breast size and volume of resected specimen, each triangle length ranged from 3 to $7 \mathrm{~cm}$, fig. $1 a, b$.

Breast lumpectomy, including skin, subcutaneous fat and glandular breast parenchyma, was achieved by excision of the tissue block., accompanied by labelling of specimen by stitching of strings of various sizes and styles. Specimen was then sent for a frozen section examination to determine the surgical margins of the tumour and also to confirm the diagnosis, fig. $2 a, b$.

A separate axillary incision was used to dissect lymph nodes in patients with invasive carcinoma. Wound closure was performed with Vicryl 2/0 for 


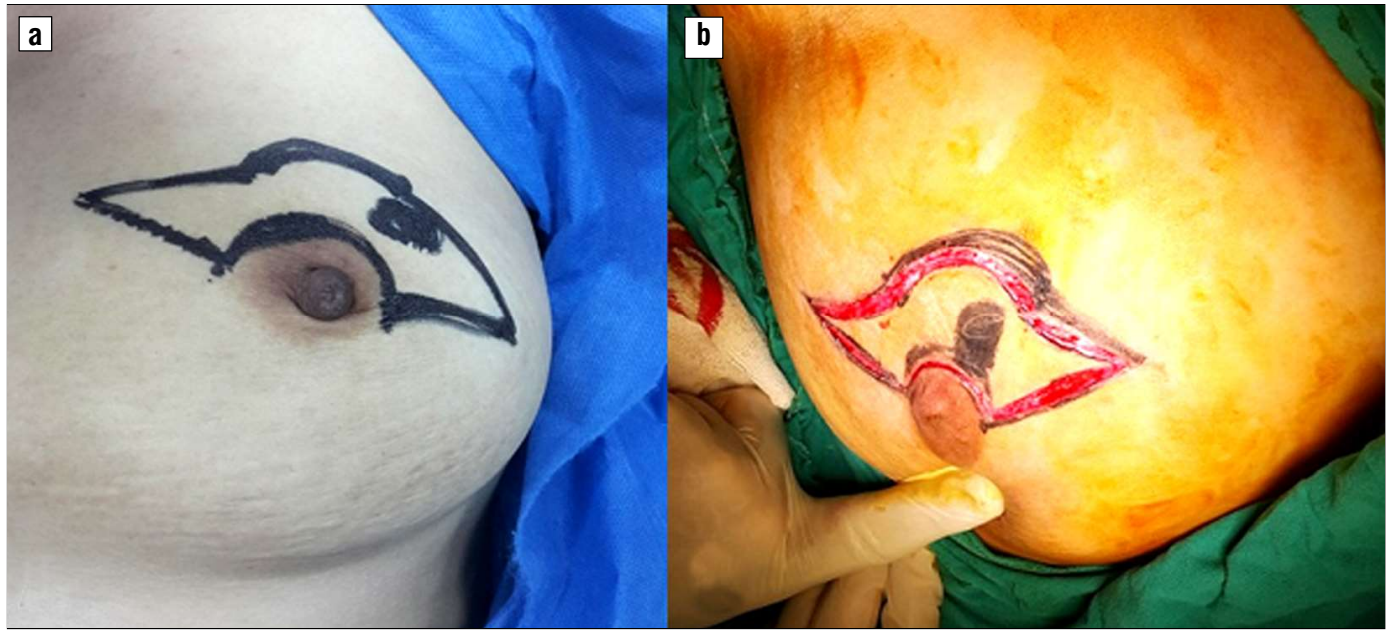

Figure 1 - (a) Preoperative marking and (b) Batwing incision

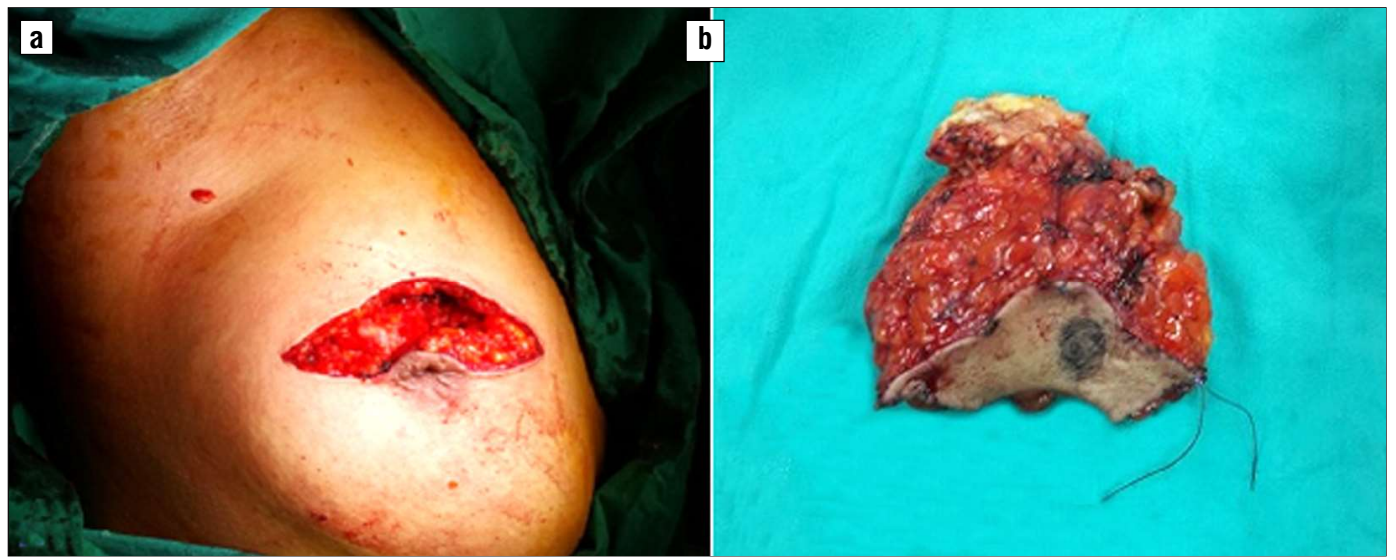

Figure 2 - (a) Lumpectomy completed and (b) stitching the margins of the specimen for orientation

glandular tissue and breast parenchyma, Vicryl $3 / 0$ for subcutaneous tissue, and Prolene $3 / 0$ for the skin. The axillary wound was closed using Vicryl $3 / 0$ for the subcutaneous tissue and Prolene $3 / 0$ for the skin

Two tube drains (one for the breast and the other for the axilla) were inserted before the skin closure through a separate incision, fig. 3. When the output was equal to or less than $30 \mathrm{~mL}$ per day, the drains were taken out. In the case of a higher amount of drainage, the drain was removed after a period of 7 days, irrespective of its output, in order to prevent surgical site infection.

The excised specimen containing tumor and/or LNs was sent for histopathology in our pathology department in TUH including ER, PR and Her-2-neu receptors. After histopathology, specimen discarded according to the conditions of ministry of health.

Post-operative follow-up: the first dressing was scheduled on the 3rd post-operative day, mainly reviewing surgical incision, local hygiene, hematomas, dehiscence, seromas (both clinically as a lump or swelling annoying the patient and by ultrasound), or wound infection.

The cosmetic result was objectively assessed by two of the authors after 4 months and scored using ABNSW

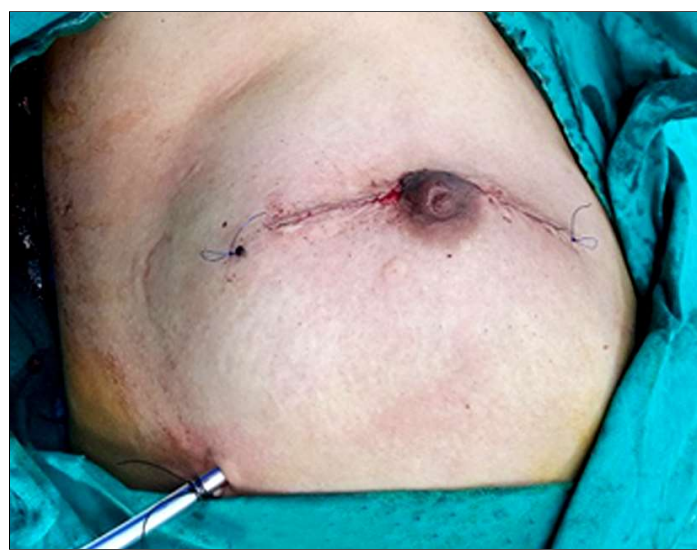

Figure 3 - After skin suturing and suction drain insertion 


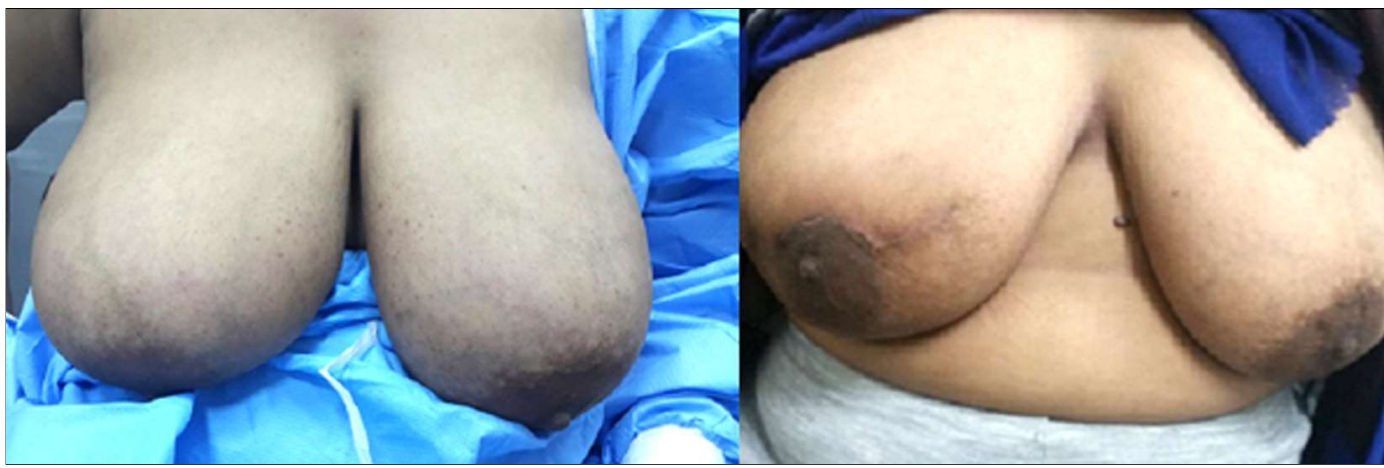

Figure 4 - Cosmetic result; preoperative and 4 months postoperative

system (9), fig. 4. Five elements are included in the ABNSW: asymmetry (A), breast shape (B), deformation of the nipple $(N)$, skin condition $(S)$ and wound scar (W). The score is scored as follows in each category: 3-excellent: there was no obvious difference between the breasts at first sight; 2-good: there were few differences between the bilateral breasts, and these were only noticeable from close observation.; 1-fair: there were obvious marked differences between the bilateral breasts; 0 poor: there were substantial, ugly differences between the bilateral breasts. We scored all five items from 0 to 3 to produce the total score. The cosmetic outcome was regarded as excellent when total score was 15 points, good when it was 11 to 14 , fair when it was 6 to 10 and poor when it was 0 to 5 .

Patients were also asked to rate their degree of satisfaction stating how they are happy with the breast shape after surgery (good, fair, and poor).

\section{RESULTS}

The ages of patients ranged from 30 to 60 years with a mean age (47.84 \pm 7.48$)$ as shown in table 1 .

Preoperatively, every patient was subjected to full history taking with special emphasis on co-morbidities and risk factors. Eleven patients had different comorbidities; three of which had more than one comorbidity as shown in table 2. The highest risk was oral contraceptive pills (16\%) followed equally by nulliparous and positive family history ( $8 \%$ ) each.

Fourteen patients (56\%) presented with left breast mass (table 3). Intraoperative frozen section was performed for all excised specimens for every patient. Safety margin was achieved in 23 patients (92\%), while the deep margin was infiltrated with malignant cells and free margins couldn't be achieved in only 2 patients (8\%) necessitating mastectomy, accordingly they were subsequently excluded (table 3 ).
Table 1 - Distribution of patients according to age

\begin{tabular}{|c|c|c|}
\hline Age Group & Number (N) & $\%$ \\
\hline$<45$ Years & 10 & 40.00 \\
\hline $45-50$ Years & 5 & 20.00 \\
\hline$>50$ Years & 10 & 40.00 \\
\hline Median & & \\
\hline
\end{tabular}

Table 2 - Co-morbidities and risk factors

\begin{tabular}{lcc}
\hline Risk factors & N & $\%$ \\
\hline Nulliparous & 2 & 8 \\
\hline Oral contraceptive pill> 8 years & 4 & 16 \\
\hline Positive family history & 2 & 8 \\
\hline Early menopause & 1 & 4 \\
\hline Comorbidities & 6 & \\
\hline Diabetes mellitus & 6 & 24 \\
\hline Hypertension & 1 & 24 \\
\hline Liver cirrhosis & 4 \\
\hline
\end{tabular}

Table 3 - Pathological findings

\begin{tabular}{|c|c|c|}
\hline Tumor location & $\mathbf{N}$ & $\%$ \\
\hline Right side & 11 & 44 \\
\hline Left side & 14 & 56 \\
\hline \multicolumn{3}{|c|}{ Pathological tumor size $(\mathrm{cm})$} \\
\hline Min. - Max. & \multicolumn{2}{|c|}{$1.0-3.0$} \\
\hline Mean \pm SD & \multicolumn{2}{|c|}{$2.01 \pm 0.56$} \\
\hline Margin & $\mathbf{N}$ & $\%$ \\
\hline Negative & 23 & 92 \\
\hline Positive & 2 & 8 \\
\hline Histology & $\mathrm{N}$ & $\%$ \\
\hline Invasive Carcinoma & 16 & 69 \\
\hline Ductal carcinoma in situ & 5 & 20 \\
\hline Fibroadenosis with sever dysplasia & 2 & 8 \\
\hline
\end{tabular}




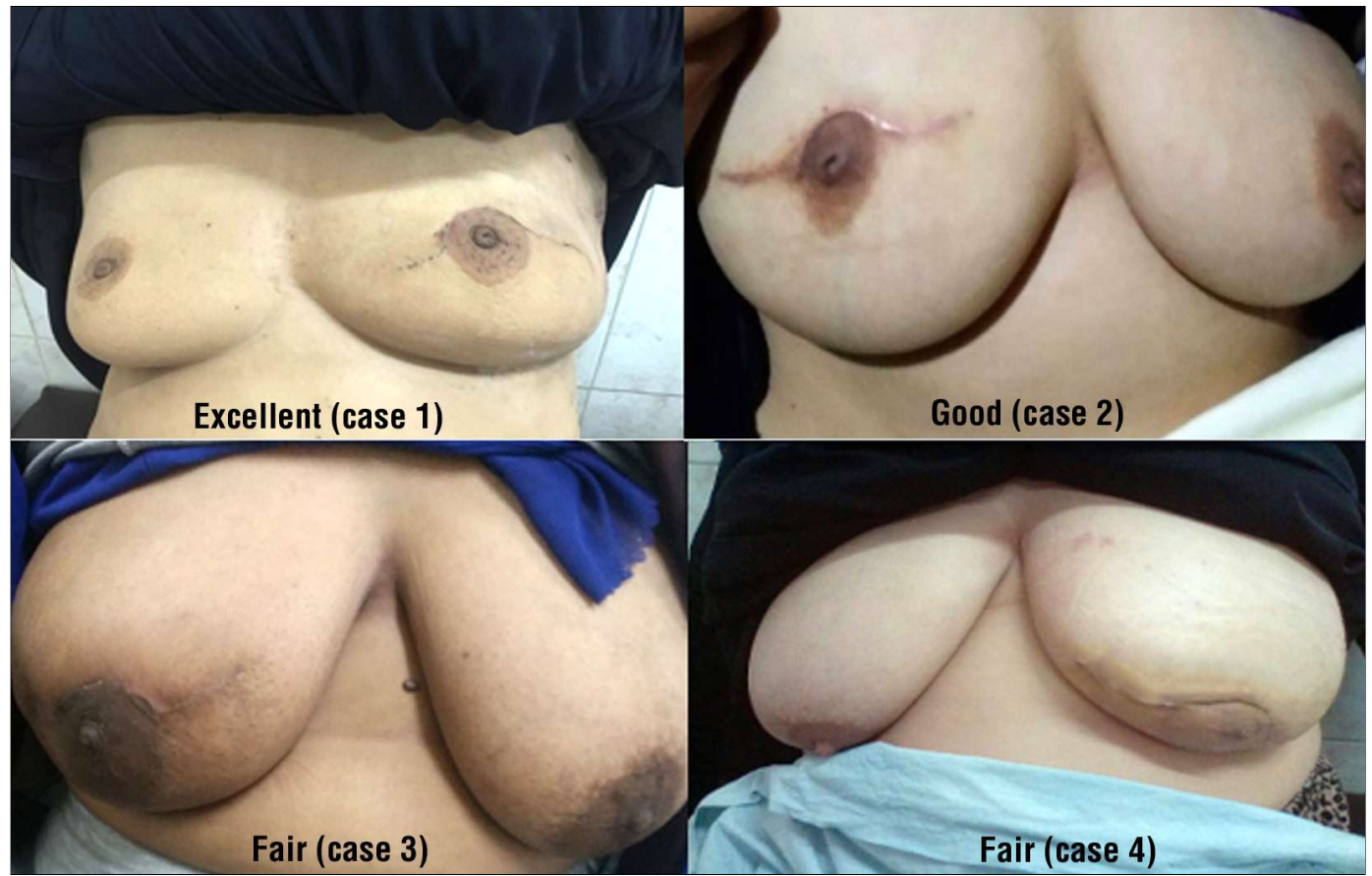

Figure 5 - 6 months post-operative cosmetic results according to ABNSW scale

The results of final histopathological examination for the resected specimens of the 23 studied patients included: most cases (16 cases) (69\%) were invasive carcinoma and 2 cases (8\%) fibroadenosis with sever dysplasia, despite they were diagnosed pre-operatively severe dysplasia and highly suspicious for malignancy (table 3).

The mean operative time was $69 \pm 14.05$ minutes, with a minimum 46 minutes and a maximum 100 minutes. Minor complications occurred in 5 patients (21.7\%); one case was complicated with breast seroma that was detected clinically and confirmed by $\mathrm{U} / \mathrm{S}$ and was reabsorbed spontaneously over 2 weeks. Two cases were complicated by axillary seroma that absorbed after 10 days. Wound infection occurred in one case and simply resolved by $3^{\text {rd }}$ generation cephalosporin, and breast hematoma occurred in one case that resolved gradually over one week (table 4).

Cosmetic outcome was evaluated 4 months post

Table 4 - Complications among the studied cases

\begin{tabular}{lcc}
\hline Complications & $\mathbf{N}$ & $\%$ \\
\hline Breast hematoma & 1 & 4.3 \\
\hline Breast seroma & 1 & 4.3 \\
\hline Axillary seroma & 2 & 8.6 \\
\hline Wound infection & 1 & 4.3 \\
\hline
\end{tabular}

operatively objectively by the operating surgeons in 23 cases according to ABNSW Scale. The result was rated as poor, fair, good or excellent, that revealed good result in 18 cases (78\%) followed by fair in 4 cases (17\%) as shown in table (5), fig. 5.

All the 23 patients were asked to rate their degree of satisfaction. The results were graded into good, fair and poor satisfaction. Eighteen patients rated good, and only 5 cases rated fair (21\%) because they were unhappy of NAC position (table 6).

Table 5 - Cosmetic outcome according to ABNSW scale (9)

\begin{tabular}{lcc}
\hline Cosmetic outcome & $\mathbf{N}$ & $\%$ \\
\hline Excellent & 1 & 4.35 \\
\hline Good & 18 & 78.26 \\
\hline Fair & 4 & 17.39 \\
\hdashline Poor & 0 & 0 \\
\hline
\end{tabular}

Table 6 - Patient satisfaction according to modified calabrese scale (10)

\begin{tabular}{lcc}
\hline Patient satisfaction & No. & $\%$ \\
\hline Good & 18 & 78.2 \\
\hline Fair & 5 & 21.7 \\
\hline Poor & 0 & 0 \\
\hline
\end{tabular}




\section{DISCUSSION}

BCS is regarded as the complete removal of breast tumors (invasive breast cancer or in situ ductal carcinoma) with a cosmetically appropriate surrounding margin of normal tissue complemented by radiation therapy (11). It has been recognized as one of the standard treatments for early-stage breast cancer (12). BCS accompanied by radiation therapy is equivalent to total mastectomy regarding local recurrence and survival rates. Long-term outcomes of randomized trials have shown that BCS produces the same oncological outcomes as mastectomy for small breast cancer (13). The main disadvantage of BCS is adverse cosmetic effects that can be observed in up to $30 \%$ of patients. The resulting breast deformities depend on several factors, such as the amount of breast tissue removed, the size of the breast (tumor to breast size ratio), whether or not the skin is resected with the tumor, the location of tumor in the breast, the positioning of the surgical incisions, and the sequel to postoperative radiation therapy (14). Early invasive breast cancer includes (stage I, lla or IIb). These are tumors of not more than $5 \mathrm{~cm}$ diameter, with either impalpable or palpable but not fixed lymph nodes and no proof of distant metastases (15).

The age of the studied patients in our series ranged from thirty to sixty years with a mean of 50.28 years. This is the same as the mean age of cases in the study by Kaur et al (16) In a comparative study conducted by Giacalone et al (17), the mean age of patients undergoing oncoplastic surgery was 51.3 years and that of patients who had quadrantectomy was 58.5 years indicating that younger age groups are more concerned about the cosmetic outcome. Six of the studied patients were diabetic, hypertensive or suffering from liver cirrhosis. Kijima et al reported one diabetic patient and another cardiac patient among their series of 7 patients (18).

Regarding the risk factors for breast cancer, in our study there were 2 nulliparous patients (8\%), 4 patients (16\%) on oral contraceptive pills, 1 patient (4\%) was early menopause and 2 patients (8\%) with positive family history. This was consistent with the study of Matkowski et al (8) on 35 patients who recorded 2 (5\%) patients with positive family history and 9 (25\%) patients out of 35 on hormone replacement therapy. Kijima et al (18) reported only one (14\%) nulliparous patient from 7 patients in their study.

Intraoperative frozen section analysis was done for the resected specimens of all patients. Free margins were assured in 23 out of the 25 patients. Of those 23 patients, the deep margin of the excised specimen was infiltrated in one patient and a re-excision was done to obtain free margins. Unfortunately, free margins couldn't be achieved in two patients for whom mastectomy was mandatory. Similar results were recorded by Dogan et al (19) who had one out of 47 patients having mastectomy for resection margin positivity. Also, our results are similar to Down et al (20) who reported re-excision of the surgical margins in one patient and required mastectomy in another patient from 37 cases. Tumor-free margins were ultimately obtained in a series of 35 cases published by Matkowski et al (8).

The mean operative time in our study was $69 \pm 14.05$ minutes with a minimum 46 minutes and a maximum 100 minutes. This was comparable to the study of Matkowski and co-workers 8 as they recorded a mean operative time of $62.7 \pm 14.4$ minutes, ranging from 40 to 103 minutes. From the histopathological point of view, among our 23 cases, 18 cases (72\%) were diagnosed as invasive ductal carcinoma and 5 cases (20\%) as DCIS. Despite that $2(8 \%)$ of the studied cases showed severe dysplasia and highly suspicious for malignancy by FNAC, their post-operative histopathology revealed fibroadenosis with severe dysplasia meaning that FNAC is not $100 \%$ reliable. Again, comparable to Matkowski et al, $880 \%$ of their patients (28 patients) had invasive ductal carcinoma and 20\% (7 patients) had DCIS. Complications-wise, one of our patients (4\%) was complicated with breast seroma, two with axillary seroma (8\%) and one diabetic patient with wound infection. Hematoma occurred in one cirrhotic patient (4\%). They all have been treated conservatively. Matkowski et al 8 similarly reported one case with breast seroma (3\%) and another one with axillary seroma (3\%).

The cosmetic appearance in our series was scored according to both surgeon and patient satisfaction taking into consideration our attempt to preserve the normal breast shape in spite of the volume of resection obtained with wider free resection margins. The overall cosmetic outcome was evaluated after 4 months objectively by operators and scored using the ABNSW system (9). Most of our patients were in the middle age group; $40 \%$ between thirty to forty-five years and $20 \%$ were between forty-five to fifty years, so cosmetic outcome was a main concern for them. Most of our patients (eighteen cases 78\%) had good cosmetic result. When patients were asked to rate their satisfaction [inspired by calabrese scale (10)], eighteen of them $(78.2 \%)$ rated good. So, most of them were really satisfied by preserving their breasts irrespective 
of any minor deformity as they kept their body image and feminizing character with complete removal of the tumor. Similarly, in Matkowski et al 8 series of 35 patients, they recorded no poor ratings of cosmetic outcome and 30 cases rated good (86\%). In spite of not using a computerized tool to assess the cosmetic outcome, we tried to be objective in recording our findings which showed that batwing mastopexy resulted in good cosmesis when performed for tumors in upper quadrants, irrespective of the side.

Our study has some constraints, firstly, it's an observational study focused on a relatively limited series of patients. Neither an historical cohort of patients nor a control group managed with other oncoplastic techniques were compared. Thus, we have to be cautious in any drawn conclusions. Besides, only short-term outcomes have been assessed and a longer follow-up is required to determine late cosmetic results. In the meantime, our single-center experience suggests that batwing mastopexy is an exciting surgical choice for periareolar tumours leading to a positive cosmetic outcome.

\section{CONCLUSION}

In conclusion, we believe that the batwing mastopexy (BWM) is a secure, accepted, convenient-tolearn and not time-consuming technique. It enables favourable breast shape and NAC position to be achieved, particularly in women who have large breasts.

\section{Conflict of interests}

The authors declare no conflicts of interest.

\section{Financial disclosure statement}

The authors declare no funding was received for this study.

\section{Ethical approval}

For performing this study ethical approval was obtained.

\section{REFERENCES}

1. Zucca Matthes AG, da Costa Viera RA, Depieri Michelli RA, Fabri
Pereira Ribeiro GH, Antônio Bailăo Jr, Raphael Luis Haikel, et al. The development of an Oncoplastic Training Center - OTC. Int J Surg. 2012;10(5):265-9.

2. van Dongen JA, Voogd AC, Fentiman IS, Legrand C, Sylvester RJ, Tong $\mathrm{D}$, et al. Long-term results of a randomized trial comparing breast-conserving therapy with mastectomy: European Organization for Research and Treatment of Cancer 10801 trial. J Natl Cancer Inst. 2000.19;92(14):1143-50.

3. Fisher B, Jeong J-H, Anderson S, Bryant J, Fisher ER, Wolmark N. Twenty-five-year follow-up of a randomized trial comparing radical mastectomy, total mastectomy, and total mastectomy followed by irradiation. N Engl J Med. 2002;347(8):567-75.

4. Fajdic J, Djurovic D, Gotovac N, Hrgovic Z. Criteria and Procedures for Breast Conserving Surgery. Acta Inform Med. 2013:21(1):16-9.

5. Holmes DR, Schooler W, Smith R. Oncoplastic approaches to breast conservation. Int J Breast Cancer. 2011;2011:303879.

6. Malter W, Kirn V, Mallmann P, Kraemer S. Oncoplastic breast reconstruction after IORT. Transl Cancer Res 2014;3(1):74-82

7. Fitzal F, Nehrer G, Deutinger M, Jakesz R, Gnant M. Novel strategies in oncoplastic surgery for breast cancer: immediate partial reconstruction of breast defects. Eur Surg. 2007;39:330-339.

8. Matkowski R, Szynglarewicz B, Kasprzak P, Forgacz J, Skalik R, Zietek M, et al. Batwing mastopexy as oncoplastic surgical approach to periareolar tumors in upper quadrants. Tumori. 2012;98(4): 421-7.

9. Yamashita K. Cosmetic Assessment. J Japan Society for Endoscopic Surgery. 2004;10(2):165-170.

10. Heil J, Carolus A, Dahlkamp J, Golatta M, Domschke C, Schuetz F, et al. Objective assessment of aesthetic outcome after breast conserving therapy: subjective third party panel rating and objective BCCT.core software evaluation. Breast. 2012;21(1):61-5.

11. Skandalakis L, Colborn G, Weidman T. Breast. In: Skandalakis' surgical anatomy. $15^{\mathrm{th}} \mathrm{Ed}$, Edited by; Skandalakis L., Colborn G. and Weidman T. McGraw-Hills. 2006. Ch. 3:151-205.

12. Malka I, Villet R, Fitoussi A. Oncoplastic conservative treatment for breast cancer. Generalities and techniques for the external quadrants. J Visc Surg. 2010;147(4):e233-7.

13. Munhoz A, Montag E, Arruda E, Pellarin L, Filassi JR, Piato JR. Assessment of immediate conservative breast surgery reconstruction: a classification system of defects revisited and an algorithm for selecting the appropriate technique. Plast Reconstr Surg. 2008; 121(3):716-727.

14. Fitzal F. Breast cancer surgery: standard principles. In: Oncoplastic Breast Surgery: A Guide to Clinical Practice, 1st Ed. Edited by; Fitzal F. and Shrenk B. Springer Science + Business Media Austria. 2010; Ch. 1: 9-2.

15. Schondorf N. Oncoplastic surgery: breast conserving surgery and immediate reconstruction: local technique. In: Oncoplastic Surgery: A Guide to Clinical Practice, 1st Ed. Edited by; Fitzal F. and Schrenk P. Springer Science + Business Media, Austria. 2010. Ch. 2:107-113.

16. Kaur N, Petit J, Rietiens M, Maffini F, Luini A, Gatti G, et al. Comparative study of surgical margins in oncoplastic surgery and quadrantectomy in breast cancer. Ann Surg Oncol. 2005;12(7): $539-45$.

17. Giacalone PL, Dubon O, Roger P, El Gareh N, Rihaoui S, Daurés JP. Doughnut mastopexy lumpectomy versus standard lumpectomy in breast cancer surgery: a prospective study. Eur J Surg Oncol. 2007; 33(3):301-6.

18. Kijima $Y$, Yoshinaka $H$, Shinden $Y$, Hirata M, Nakajo A, Arima $H$, et al. Oncoplastic breast surgery for centrally located breast cancer: a case series. Gland Surg. 2014;3(1):62-73.

19. Dogan L, Gulcelik MA, Karaman N, Ozaslan C, Reis E. Oncoplastic surgery in surgical treatment of breast cancer: is the timing of adjuvant treatment affected? Clin Breast Cancer. 2013;13(3):202-5.

20. Down SK, Jha PK, Burger A, Hussien MI. Oncological advantages of oncoplastic breast-conserving surgery in treatment of early breast cancer. Breast J. 2013;19(1):56-63. 